\title{
Amalgam Tattoo
}
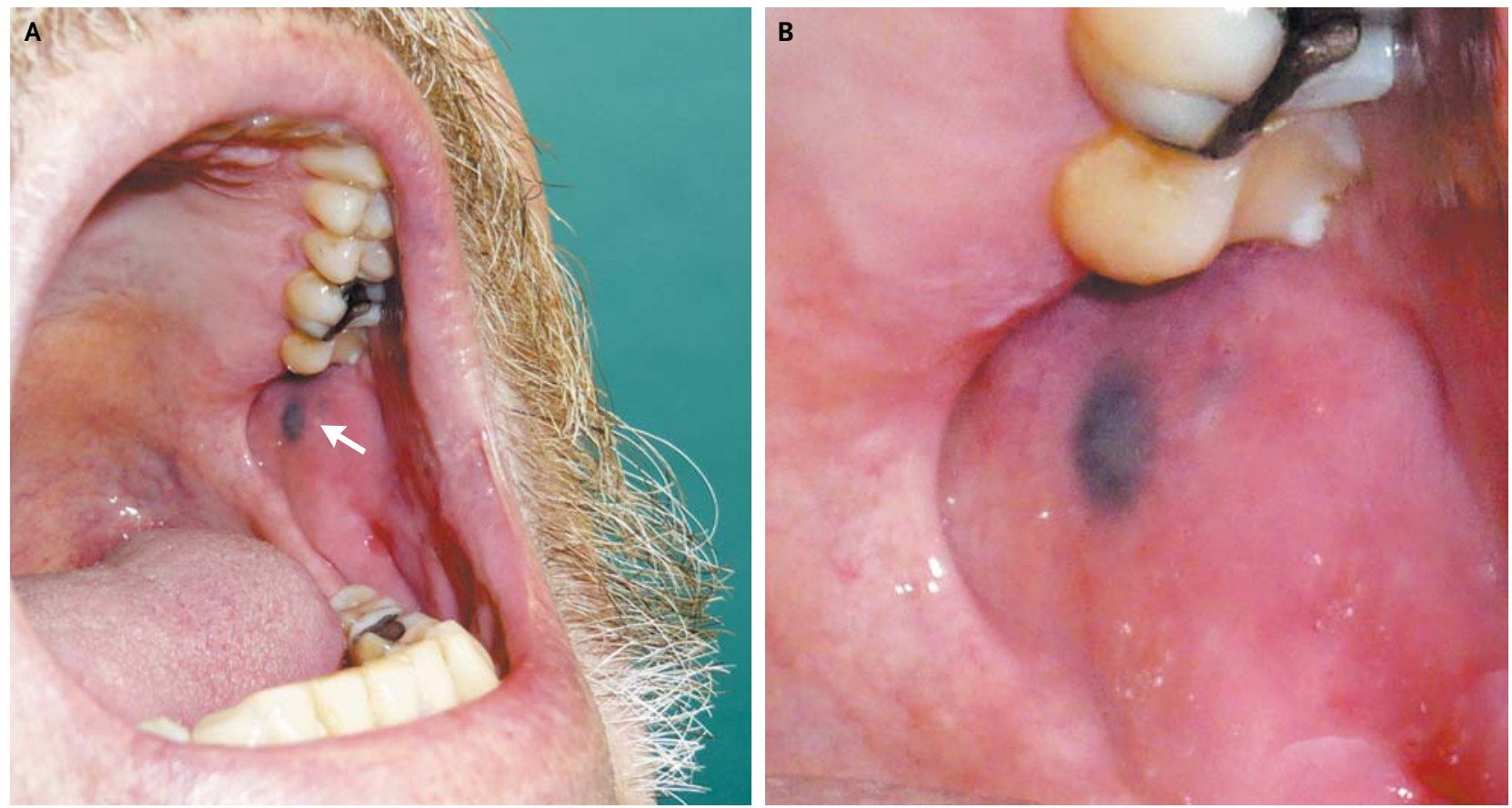

64-YEAR-OLD MAN WAS REFER RED FOR EVALUATION OF AN ASYMPTOMATIC pigmented lesion on the buccal mucosa (Panels A and B). After 15 pack-years of smoking, he had quit smoking more than 30 years earlier and had no other risk factors for oral cancer. A biopsy of the lesion revealed interstitial accumulation of brown and black particles within the submucosa, a finding that was compatible with a diagnosis of amalgam tattoo. Amalgam dental fillings contain mercury, silver, and other metals that may inadvertently be implanted into adjacent gingival, buccal, palatal, or lingual mucosa at the time of tooth restoration. This produces black, blue, or gray macules that are asymptomatic and change little over time. Although amalgam tattoos are common and benign, they may mimic pigmented oral lesions with more worrisome causes, such as melanoma or Kaposi's sarcoma. Only a minority of amalgam tattoos are radioopaque on plain radiography. A biopsy can be performed without difficulty and is effective in ruling out cancer, although it is usually unnecessary when the lesion is typical and has been evaluated by an experienced practitioner.

Copyright (c) 2011 Massachusetts Medical Society.
Patrick Dubach, M.D.

Marco Caversaccio, M.D.

Department of Otorhinolaryngology, Head and Neck Surgery, University of Bern Inselspital

Bern, Switzerland patrickdubach@insel.ch 